\title{
Mecanismos de regeneração natural em diferentes ambientes de remanescente de Floresta Ombrófila Mista, São Francisco de Paula, RS
}

\author{
Mechanisms of natural regeneration in different environments in the remaining Mixed Rain Forest, \\ São Francisco de Paula, RS
}

\author{
Luciane Belmonte Chami ${ }^{*}$ Maristela Machado Araujo ${ }^{\mathrm{I}}$ Solon Jonas Longhi ${ }^{\mathrm{I}}$ Paula Kielse $^{\mathrm{I}}$ \\ Alessandro Dal'Col Lúcio ${ }^{\mathrm{II}}$
}

\section{RESUMO}

\begin{abstract}
O objetivo deste trabalho foi avaliar os mecanismos de regeneração natural em três agrupamentos florísticos em remanescente de Floresta Ombrófila Mista, localizado em São Francisco de Paula, RS. A coleta dos dados foi realizada em seis conglomerados de um hectare, subdivididos em 16 parcelas cada, nas quais foram coletadas amostras da chuva de sementes, do banco de sementes no solo e do banco de plântulas. Os grupos florísticos apresentaram diferenças significativas para a densidade de indivíduos na chuva de sementes e no banco de plântulas. Para o banco de sementes no solo, o grupo $G_{1}$ diferiu dos grupos $G_{2}$ e $G_{3}$. A riqueza de espécies observada no banco de plântulas sugere que este pode ser o mecanismo principal de manutenção da diversidade no remanescente. A chuva de sementes foi abundante $e$ representou uma fonte expressiva de propágulos para abastecer o banco de sementes e banco de plântulas local e das áreas circunvizinhas. $O$ banco de sementes do solo não apresentou potencial florístico para representar a riqueza de espécies presentes na vegetação arbórea, corroborando o estágio sucessional avançado desta floresta, uma vez que o banco de sementes é formado principalmente de espécies pioneiras.
\end{abstract}

Palavras-chave: chuva de sementes, banco de sementes, banco de plântulas, agrupamentos.

\section{ABSTRACT}

The aim of this study was to evaluate the natural regeneration mechanisms in three floristic groups of the remaining Mixed Rain Forest, located in San Francisco de Paula, RS. The data were collected in six conglomerates of one hectare, subdivided into 16 plots, in which seed rain, soil seed bank, and seedling bank were collected. The groups presented differences in the density of individuals in the seed rain and in the seedling banks. For the soil seed bank, the group $G_{I}$ differed from the groups $G_{2}$ and $G_{3}$. The seedlings bank, due to the wealth of species, may be the primary mechanism for maintaining diversity in the remnant. Seed rain was abundant and represented an expressive source of seeds to supply the seed bank and the seedling bank site and surrounding areas. The seed bank of soil did not show potential to replace the floristic richness of species in the arboreal vegetation, confirming the advanced stage of forest succession, once the seed bank is composed mainly of pioneer species

Key words: seed rain, soil seed bank, seedling banks, groups.

\section{INTRODUÇÃO}

A Floresta Ombrófila Mista é uma tipologia formada pela mistura de floras de diferentes origens que define um padrão fitofisionômico típico da região sul do Brasil, o Planalto Meridional Brasileiro (LEITE \& KLEIN, 1990). No Rio Grande do Sul, as florestas naturais remanescentes constituem laboratórios naturais importantes à pesquisa, fonte de informações fitosociológicas, ecológicas e silviculturais necessárias para restauração, recuperação e manejo de áreas de mesma formação fitoecológica.

A diversidade de espécies encontradas na forma de sementes dispersadas, dormentes no solo ou plântulas, indica o estado de conservação do ecossistema e a sua capacidade de auto-regeneração ou resiliência, cujo grau pode determinar a necessidade

'Departamento de Ciências Florestais, Universidade Federal de Santa Maria (UFSM), 97105-900, Santa Maria, RS, Brasil. E-mail: lchami@terra.com.br. *Autor para correspondência.

"Departamento de Fitotecnia, UFSM, Santa Maria, RS, Brasil. 
de intervenção humana. A chuva de sementes corresponde à dispersão das sementes e a área abrangida por esse processo até o estabelecimento da plântula (ALMEIDA-CORTEZ, 2004). O banco de sementes é formado por sementes viáveis presentes no solo, em um dado momento, desconsiderando a época de sua entrada no ambiente (HYATT, 1999). A formação do banco de plântulas ocorre após a produção, dispersão e germinação das sementes, fase caracterizada pela elevada densidade de indivíduos.

Na Floresta Nacional (FLONA) de São Francisco de Paula, o Projeto Ecológico de Longa Duração (PELD) desenvolve diversos estudos abordando crescimento, estrutura e dinâmica da vegetação em Floresta Ombrófila Mista, contudo, pouco se conhece sobre as características específicas dos mecanismos de regeneração. Muitos trabalhos relacionados à estrutura e dinâmica da vegetação consideram a existência de grupos de espécies arbóreas, com características biológicas e ecológicas comuns e que, por isso, utilizam os recursos do ambiente de maneira semelhante (FINEGAN \& SABOGAL, 1988). CHAMI (2008), estudando a vegetação arbórea da Floresta Ombrófila Mista, na FLONA de São Francisco de Paula, demonstrou por análise multivariada a formação de três grupos florísticos, caracterizados por espécies e ambientes específicos.

Considerando as diferentes estruturas populacionais presentes no remanescente de Floresta Ombrófila Mista na FLONA, o objetivo deste estudo foi avaliar os mecanismos de regeneração das espécies arbóreas e arbustivas nos agrupamentos florísticos, enfocando o potencial de cada mecanismo de regeneração na dinâmica da floresta.

\section{MATERIAL E MÉTODOS}

O estudo foi realizado na FLONA de São Francisco de Paula, localizada na serra gaúcha, entre as coordenadas geográficas $29^{\circ} 24^{\prime}$ e $29^{\circ} 27^{\prime} \mathrm{S}$ e $50^{\circ} 22^{\prime}$ e $50^{\circ} 25^{\prime} \mathrm{W}$, ocupando uma área total de 1.606,69 ha; destes 901,9 ha (56\%) são cobertos com floresta natural. A região apresenta valor médio anual de temperatura, no mês mais quente, de $22^{\circ} \mathrm{C}$ e, no mês mais frio, temperaturas entre $-3^{\circ} \mathrm{C}$ a $18^{\circ} \mathrm{C}$ (MORENO, 1961). A precipitação é bem distribuída em todos os meses do ano, com média anual de 2.252mm (NIMER, 1990). O solo é do tipo Cambissolo Húmico Alumínico típico (EMBRAPA, 1999). A vegetação constitui um mosaico de formações que compreende remanescentes de Floresta Ombrófila Mista, florestas de transição, savanas, reflorestamentos de Eucalyptus spp., Pinus spp., Cryptomeria japonica (Thunb. ex L. f.) D. Don e Araucaria angustifolia. A floresta nativa caracterizase pela presença de A. angustifolia, associada com Blepharocalyx salicifolius, Campomanesia xanthocarpa, Cryptocarya aschersoniana, Dicksonia sellowiana, Ilex paraguariensis, Ilex brevicuspis, Lithraea brasiliensis Marchand, Matayba elaeagnoides, Myrcianthes gigantea, Nectandra megapotamica, entre outras (RIO GRANDE DO SUL, 2001).

Na FLONA de São Francisco de Paula, encontram-se distribuídos dez conglomerados de um hectare, que constituem o Projeto Ecológico de Longa Duração (PELD). A coleta de dados foi realizada em seis desses conglomerados, selecionados adotandose como critérios: variações de declividade, posição topográfica, características físicas do solo e exposição do terreno. Cada conglomerado foi subdividido em 16 parcelas de 20x20m, mantendo-se 10m de entorno. Para a avaliação da chuva de sementes, do banco de sementes do solo e do banco de plântulas foram considerados os seguintes agrupamentos: $G_{1}-$ encosta; $\mathrm{G}_{2}$ - árvores emergentes (dossel dominado por indivíduos de grande porte de A. angustifolia); e $\mathrm{G}_{3}$-úmido (CHAMI, 2008).

Para o estudo da composição e densidade da chuva de sementes, foram instalados sistematicamente, no centro de cada parcela de 20x20m, 96 coletores circulares $\left(1 \mathrm{~m}^{2}\right)$ equidistantes $20 \mathrm{~m}$, dispostos a $1 \mathrm{~m}$ da superfície do solo. O material vegetal depositado nos coletores foi recolhido mensalmente, durante o período de janeiro a dezembro de 2007 e conduzido ao Laboratório de Silvicultura da Universidade Federal de Santa Maria (UFSM). As sementes maiores que $1 \mathrm{~mm}$ foram separadas, identificadas e quantificadas.

Para a caracterização da composição e densidade do banco de sementes no solo, realizou-se a coleta de 96 amostras de $5 \mathrm{~cm}$ de solo (descartando a serapilheira), com uso de um gabarito de ferro de $25 \times 25 \mathrm{~cm}$, em ponto adjacente aos coletores de chuva de sementes. As amostras foram conduzidas à casa de vegetação e depositadas em bandejas $(30 \times 40 \mathrm{~cm})$ preenchidas com $3 \mathrm{~cm}$ de vermiculita. As bandejas foram distribuídas aleatoriamente sobre bancadas e cobertas com sombrite (50\%) para evitar a entrada de propágulos externos. O experimento foi conduzido por oito meses, com irrigação e monitoramento diários. Os indivíduos (árvores e arbustos) foram mensalmente identificados e quantificados.

A avaliação do banco de plântulas foi realizada em 72 unidades amostrais de $2 \times 2 \mathrm{~m}$, distribuídas sistematicamente de $20 \mathrm{em} 20 \mathrm{~m}$, na mesma 
linha dos coletores de chuva de sementes. O banco de plântulas foi representado pelos indivíduos com altura $\geq 20 \mathrm{~cm}$ e diâmetro à altura do peito (DAP) $\leq 1 \mathrm{~cm}$, considerando-se três classes de tamanho $(\mathrm{Ct}):(1)-\mathrm{Ct}$ I: de 20 a $50 \mathrm{~cm}$ de altura; (2) - Ct II: de 51 a $100 \mathrm{~cm}$ de altura; (3) - Ct III: $>100 \mathrm{~cm}$ de altura e DAP $=1 \mathrm{~cm}$.

Os dados de densidade média de indivíduos não apresentaram distribuição normal, então foram analisados pelo teste não-paramétrico da Soma das Ordens de Wilcoxon (W) (CARNELUTTI FILHO et al., 2001). A suficiência amostral do banco de plântulas foi obtida pelo método da curva espécie/área (BRAUNBLANQUET, 1979).

\section{RESULTADOS E DISCUSSÃO}

No estudo de chuva de sementes, foram amostradas 55 espécies: $56 \%$ identificadas e $44 \%$ classificadas apenas como morfo-espécie. A dificuldade na identificação das espécies por meio das sementes também ocorreu com VIEIRA (1996). Myrtaceae, Fabaceae, Aquifoliaceae, Lauraceae e Euphorbiaceae foram as famílias que apresentaram o maior número de espécies (Tabela 1 e 2).

O teste de Wilcoxon demonstrou que o G1 apresentou maior densidade média de sementes, diferindo dos G2 e G3, que também diferiram entre si (Figura 1A). A diferença foi indicada pelos valores de Wcalculado $=\mathrm{Z}$ tabelado $(\mathrm{P}<0,05)(\mathrm{Z}$ tabelado $=1,96)$ $\left(\mathrm{W}_{\mathrm{G} 1 \times \mathrm{G} 2}=4,81 ; \mathrm{W}_{\mathrm{G} 1 \times \mathrm{G} 3}=2,09 ; \mathrm{W}_{\mathrm{G} 2 \times \mathrm{G} 3}=3,21\right)$.

A elevada densidade de sementes no $\mathrm{G}$ foi ocasionada pela dispersão de Vernonia discolor, observada em $100 \%$ das unidades amostrais. Essa espécie tem comportamento pioneiro, apresenta elevada produção de sementes pequenas e leves, sendo comumente encontrada em sub-bosque da mata de araucária que sofreu interferência humana (LORENZI, 2002). Juntamente com $\boldsymbol{V}$. discolor, as espécies mais representativas na chuva de sementes no $\mathrm{G}_{1}$ foram Ilex microdonta, Lamanonia ternata, Ilex paraguariensis, Myrsine laetevirens, Ilex brevicuspis e Sebastiania brasiliensis (Tabela 3 ). $\mathrm{O} \mathrm{G}_{2}$ foi o local com a menor entrada de diásporas, em que I. paraguariensis, $\boldsymbol{I}$. brevicuspis e $\boldsymbol{S}$. brasiliensis contribuíram com cerca de $90 \%$ da chuva de sementes. Araucaria angustifolia, classificada como espécie pioneira, teve baixa produção de sementes mesmo predominando no estrato arbóreo do grupo. Isso, associado à apreciação do pinhão pela fauna, ausência de dormência e comportamento recalcitrante das sementes, poderá refletir negativamente na regeneração da espécie na FLONA. Por outro lado, a característica heliófila dessa espécie sugere um alto potencial regenerativo na floresta quando a chuva de sementes coincidir com a abertura de clareiras, pois sementes recalcitrantes não permanecem viáveis por muito tempo no solo e espécies heliófilas raramente formam um banco de plântulas. Assim, destaca-se a dificuldade em classificar a $\boldsymbol{A}$. angustifolia quanto ao grupo ecológico, conforme já observado por DUARTE et al. (2002), uma vez que também apresenta aspectos típicos das espécies tardias: vida longa, fustes capazes de atingir diâmetros maiores de $60 \mathrm{~cm}$, sementes grandes com curta viabilidade(BUDOWSKI, 1965; FERRETTI, 2002). No $\mathrm{G}_{3}$, Ilex brevicuspis foi a espécie mais representativa, contribuindo com aproximadamente $90 \%$ do total das sementes dispersadas nesse grupo.

Apesar da pequena diversidade de espécies observada na chuva de sementes, a densidade média de sementes aparentemente viável foi elevada, o que demonstra uma abundante entrada de propágulos no remanescente. SCOTTI (2009) também observou alta densidade de sementes em remanescente de Floresta Estacional Decidual no Rio Grande do Sul. Já ARAUJO et al. (2004) encontraram baixa dispersão de sementes em Floresta Estacional Decidual Ripária no Rio Grande do Sul e, segundo os mesmos autores, as intensas enchentes ocorridas no período de coleta, provavelmente, afetaram os processos fisiológicos das plantas. Sendo assim, a variação do potencial da chuva de sementes, até mesmo entre as regiões de clima semelhante, pode estar mais relacionada com o grau de perturbação no ecossistema do que com a região fitoecológica.

O banco de sementes do solo demonstrou um baixo potencial de restauração da riqueza de espécies arbóreas. Foram identificadas 59 espécies de 40 gêneros e 21 famílias, sendo 38 herbáceas (dados não publicados), 14 arbóreas e sete arbustivas (Tabela 1 e 2). Esse mecanismo de regeneração é importante quando o ecossistema é submetido a distúrbios mais severos e a presença de espécies herbáceas é um bom indicador de resiliência, quando estas não são exóticas (MARTINS et al., 2008). As famílias que apresentaram o maior número de espécies foram Asteraceae, Solanaceae, Aquifoliaceae, Euphorbiaceae e Myrsinaceae.

Considerando o número de indivíduos de espécies arbóreas e arbustivas germinadas no banco de sementes dos distintos grupos, a análise estatística indicou diferença significativa entre $G_{1}$ e os grupos $G_{2}$ e $\mathrm{G}_{3}$, que não diferiram entre si (Figura $1 \mathrm{~B}$ ). No $\mathrm{G}_{1}$, a espécie melhor representada foi Ilex paraguariensis, seguida de Ilex brevicuspis, Zanthoxylum rhoifolium e Siphoneugena reitzii (Tabela 3). No $\mathrm{G}_{2}$ e $\mathrm{G}_{3}, \boldsymbol{I}$. brevicuspis apresentou a maior densidade de sementes. 
Tabela 1 - Espécies arbóreas e arbustivas presentes nos mecanismos de regeneração em remanescente de Floresta Ombrofila Mista, na FLONA de São Francisco de Paula, RS

\begin{tabular}{|c|c|c|c|c|c|c|}
\hline Família & Nome científico & GE & FV & $\mathrm{CS}$ & BS & $\mathrm{BP}$ \\
\hline Annonaceae & Rollinia rugulosa Schltdl. & $\mathrm{PS}_{\mathrm{i}}$ & 1 & & & $*$ \\
\hline \multirow[t]{4}{*}{ Aquifoliaceae } & Ilex brevicuspis Reissek & $\mathrm{S}_{\mathrm{i}}$ & 1 & $*$ & * & $*$ \\
\hline & Ilex dumosa Reissek & $\mathrm{Si}$ & 1 & $*$ & $*$ & \\
\hline & Ilex microdonta Reissek & $\mathrm{Si}$ & 1 & $*$ & & $*$ \\
\hline & Ilex paraguariensis A.St-Hil. & $\mathrm{Si}$ & 1 & $*$ & $*$ & $*$ \\
\hline Araucariaceae & Araucaria angustifolia (Bertol.) Kuntze & $\mathrm{P}$ & 1 & $*$ & & $*$ \\
\hline \multirow[t]{7}{*}{ Asteraceae } & Baccharis dracunculifolia DC. & $P$ & 2 & & $*$ & \\
\hline & Baccharis elaeagnoides Steud. ex Baker & $\mathrm{P}$ & 2 & & $*$ & \\
\hline & Baccharis sp. & $P$ & 2 & & $*$ & \\
\hline & Baccharis uncinella DC. & $\mathrm{P}$ & 2 & & $*$ & \\
\hline & Dasyphyllum spinescens (Less.) Cabrera & $\mathrm{P}$ & 1 & & & $*$ \\
\hline & Piptocarpha notata (Less.) Baker & & & & & $*$ \\
\hline & Vernonia discolor (Spreng.) Less. & $P$ & 1 & $*$ & & $*$ \\
\hline \multirow[t]{2}{*}{ Cannabaceae } & Celtis iguanea (Jacq.) Sarg. & $\mathrm{P}$ & 2 & $*$ & $*$ & \\
\hline & Celtis sp. & & 2 & $*$ & & \\
\hline \multirow[t]{2}{*}{ Celastraceae } & Maytenus aquifolia Mart. & & 1 & & & $*$ \\
\hline & Maytenus evonymoides Reissek & $\mathrm{S}_{\mathrm{t}} \mathrm{C}$ & 1 & $*$ & & $*$ \\
\hline \multirow[t]{2}{*}{ Cunoniaceae } & Lamanonia ternata Vell & $\mathrm{P}$ & 1 & $*$ & $*$ & $*$ \\
\hline & Weinmannia paulliniifolia Pohl ex Ser. & $\mathrm{PS}_{\mathrm{i}}$ & 1 & & $*$ & \\
\hline Dicksoniaceae & Dicksonia sellowiana Hook. & $\mathrm{C}$ & 1 & & & $*$ \\
\hline \multirow[t]{4}{*}{ Euphorbiaceae } & Sapium glandulosum (L.) Morong & $\mathrm{PS}_{\mathrm{i}}$ & 1 & $*$ & * & \\
\hline & Sebastiania brasiliensis Spreng. & $\mathrm{S}_{\mathrm{t}}$ & 1 & $*$ & & $*$ \\
\hline & Sebastiania commersoniana (Baill.) & $\mathrm{S}_{\mathrm{t}}$ & 1 & $*$ & $*$ & $*$ \\
\hline & Stillingia oppositifolia Baill. Ex. Müll.Arg. & $\mathrm{C}^{\mathrm{I}}$ & 1 & & & $*$ \\
\hline \multirow[t]{7}{*}{ Fabaceae } & Acacia bonariensis Gillies ex Hook. \& Arn. & $\mathrm{P}$ & 2 & $*$ & & $*$ \\
\hline & Dalbergia frutescens (Vell.) Britton & $\mathrm{P}$ & 1 & $*$ & & $*$ \\
\hline & Inga vera Willd. & $\mathrm{P}$ & 1 & & & $*$ \\
\hline & Lonchocarpus campestris Mart. Ex Benth. & $P$ & 1 & $*$ & & $*$ \\
\hline & Machaerium aculeatum Raddi & $\mathrm{P}$ & & & & $*$ \\
\hline & Machaerium paraguariense Hassl. & & 1 & & & $*$ \\
\hline & Mimosa scabrella Benth. & $\mathrm{S}_{\mathrm{i}}$ & 1 & & $*$ & \\
\hline \multirow[t]{9}{*}{ Lauraceae } & Cinnamomum amoenum (Nees) Kosterm. & & 1 & $*$ & & $*$ \\
\hline & Cinnamomum glaziovii (Mez) Kosterm. & $\mathrm{S}_{\mathrm{t}}$ & 1 & & & $*$ \\
\hline & Cryptocarya aschersoniana $\mathrm{Mez}$ & $\mathrm{S}_{\mathrm{t}}$ & 1 & $*$ & & $*$ \\
\hline & Endlicheria panicultata (Spreng.) J. F. Macbr. & $\mathrm{S}_{\mathrm{t}}$ & 1 & & & $*$ \\
\hline & Nectandra megapotamica (Spreng.) Mez & $\mathrm{S}_{\mathrm{t}}$ & 1 & $*$ & & $*$ \\
\hline & Ocotea diospyrifolia (Meisn.) Mez. & $\mathrm{S}_{\mathrm{t}}$ & 1 & & & $*$ \\
\hline & Ocotea indecora $(\mathrm{Schott}) \mathrm{Mez}$ & & 1 & & & $*$ \\
\hline & Ocotea puberula (Rich.) Nees & $\mathrm{S}_{\mathrm{t}}$ & 1 & & & $*$ \\
\hline & Ocotea pulchella (Nees) Mez & $\mathrm{S}_{\mathrm{i}}$ & 1 & $*$ & & $*$ \\
\hline Loganiaceae & Strychnos brasiliensis (Spreng.) Mart. & $\mathrm{S}_{\mathrm{i}} \mathrm{S}_{\mathrm{t}}$ & 1 & & & $*$ \\
\hline Malvaceae & Luehea divaricata Mart. \& Zucc. & $\mathrm{P}$ & 1 & $*$ & & $*$ \\
\hline Melastomataceae & Miconia sp. & & 2 & & & $*$ \\
\hline
\end{tabular}

GE: Grupo ecológico - adaptado de CHAMI (2008) e ${ }^{\mathrm{I}}$ MOSCOVICH (2006); P: pioneira; $\mathrm{S}_{\mathrm{i}}$ : secundária inicial; $\mathrm{S}_{\mathrm{t}}$ : secundária tardia; FV: formas de vida; $1=$ árvore; 2 =arbusto; CS: chuva de sementes; BS: banco de sementes do solo; BP: banco de plântulas.

Já I. paraguariensis ocorreu com baixa densidade quando comparada aos valores observados no $\mathrm{G}_{1}$. Sebastiania commersoniana, apesar de ser a espécie de maior valor de importância na vegetação arbórea (CHAMI, 2008), e da presença na chuva de sementes, apresentou baixa densidade no banco de sementes no $\mathrm{G}_{3} \mathrm{~A}$ ausência de dormência e a alta taxa de germinação das sementes de S. commersoniana (LORENZI, 2002) podem explicar a permanência transitória dessa espécie no banco de sementes solo.

Ciência Rural, v.41, n.2, fev, 2011. 
Tabela 2 - Espécies arbóreas e arbustivas presentes nos mecanismos de regeneração em remanescente de Floresta Ombrofila Mista, na FLONA de São Francisco de Paula, RS.

\begin{tabular}{|c|c|c|c|c|c|c|}
\hline Família & Nome científico & GE & FV & $\mathrm{CS}$ & BS & BP \\
\hline \multirow[t]{3}{*}{ Monimiaceae } & Hennecartia omphalandra J.Poiss. & $\mathrm{Si}$ & 1 & & & $*$ \\
\hline & Mollinedia elegans Tul. & $\mathrm{C}^{\mathrm{I}}$ & 2 & & & * \\
\hline & Mollinedia sp. & & 2 & & & * \\
\hline \multirow[t]{4}{*}{ Myrsinaceae } & Myrsine coriacea (Sw.) R.Br. & $\mathrm{Si}$ & 1 & & $*$ & $*$ \\
\hline & Myrsine laetevirens (Mez) Arechav. & $\mathrm{Si}$ & 1 & * & $*$ & \\
\hline & Myrsine sp. & & 1 & $*$ & & $*$ \\
\hline & Myrsine umbellata Mart. & $\mathrm{Si}$ & 1 & & $*$ & $*$ \\
\hline \multirow[t]{17}{*}{ Myrtaceae } & Blepharocalyx salicifolius (Kunth) O.Berg & $\mathrm{Si}$ & 1 & $*$ & & $*$ \\
\hline & Calyptranthes concinna DC. & St & 1 & & & $*$ \\
\hline & Campomanesia rhombea O.Berg & $\mathrm{Si}$ & 1 & & & $*$ \\
\hline & Campomanesia xanthocarpa O.Berg. & St C & 1 & $*$ & & $*$ \\
\hline & Eugenia involucrata DC. & $\mathrm{C}$ & 1 & & & $*$ \\
\hline & Eugenia uniflora $\mathrm{L}$. & & 1 & & & $*$ \\
\hline & Eugenia uruguayensis Cambess. & St & 1 & $*$ & & $*$ \\
\hline & Myrceugenia cucullata D. Legrand & $\mathrm{C}$ & 1 & & & $*$ \\
\hline & Myrceugenia glaucescens (Camb.) D. Legr. & & & & & * \\
\hline & Myrceugenia miersiana(Gard.) D. Legr. \& K. & $\mathrm{C}$ & 1 & & & * \\
\hline & Myrceugenia myrcioides (Cambess.) O.Berg & $\mathrm{C}$ & 1 & & & * \\
\hline & Myrceugenia oxysepala(Burr.)D.Legr.\& K. & $\mathrm{C}^{\mathrm{I}}$ & 1 & & & * \\
\hline & Myrceugenia sp. & & 1 & & & $*$ \\
\hline & Myrcia bombycina (O.Berg) Nied. & & 1 & & & * \\
\hline & Myrcia oligantha O.Berg & $\mathrm{C}$ & 1 & $*$ & & $*$ \\
\hline & Myrcianthes gigantea D.Legrand & St & 1 & & & $*$ \\
\hline & Siphoneugena reitzii D.Legrand. & $\mathrm{S}_{\mathrm{i}} \mathrm{S}_{\mathrm{t}}$ & 1 & & $*$ & $*$ \\
\hline Phytolaccaceae & Seguieria aculeata L. & & & & & * \\
\hline Picramniaceae & Picramnia parvifolia Engl. & & 1 & & & $*$ \\
\hline Piperaceae & Piper sp. & & 2 & & & * \\
\hline Podocarpaceae & Podocarpus lambertii Klotzsch ex Endl. & $\mathrm{S}_{\mathrm{i}} \mathrm{S}_{\mathrm{t}}$ & 1 & $*$ & & * \\
\hline Proteaceae & Roupala brasiliensis Klotzsch & $\mathrm{C}$ & 1 & & & * \\
\hline Rosaceae & Prunus myrtifolia (L.) Urb. & $S_{i} S_{t}$ & 1 & & & * \\
\hline Rubiaceae & Rudgea parquioides (Cham.) Müll. Arg. & $\mathrm{C}^{\mathrm{I}}$ & 2 & * & & * \\
\hline \multirow[t]{2}{*}{ Rutaceae } & Zanthoxylum petiolare A. St.Hil. e Tul. & & 1 & & & * \\
\hline & Zanthoxylum rhoifolium Lam. & $\mathrm{S}_{\mathrm{i}}$ & 1 & & $*$ & * \\
\hline \multirow[t]{2}{*}{ Salicaceae } & Casearia decandra Jacq. & $\mathrm{S}_{\mathrm{i}}$ & 1 & $*$ & & * \\
\hline & Xylosma pseudosalzmannii Sleumer & $\mathrm{S}_{\mathrm{i}}$ & 1 & & & * \\
\hline \multirow[t]{4}{*}{ Sapindaceae } & Allophylus edulis (A.St.Hil.,Camb..\& A.Juss.) Radl. & $\mathrm{S}_{\mathrm{i}}$ & 1 & & & * \\
\hline & Allophylus guaraniticus (A.St.-Hil.) Radlk & $\mathrm{S}_{\mathrm{i}} \mathrm{St}$ & 1 & & & * \\
\hline & Cupania vernalis Cambess. & $\mathrm{S}_{\mathrm{t}}$ & 1 & $*$ & & * \\
\hline & Matayba elaeagnoides Radlk. & $\mathrm{S}_{\mathrm{t}}$ & 1 & $*$ & & * \\
\hline \multirow[t]{4}{*}{ Solanaceae } & Brunfelsia uniflora (Pohl) D. Don & $\mathrm{P}$ & 2 & & & * \\
\hline & Cestrum intermedium Sendtn. & $P$ & 1 & & $*$ & $*$ \\
\hline & Solanum mauritianum Scop. & & 1 & & * & \\
\hline & Solanum sanctaecatharinae Dunal & Si St & 1 & & * & \\
\hline Symplocaceae & Symplocos uniflora (Pohl) Benth. & $\mathrm{S}_{\mathrm{i}} \mathrm{S}_{\mathrm{t}}$ & 1 & & $*$ & \\
\hline Theaceae & Laplacea acutifolia (Wawra) Kobuski & $\mathrm{P}$ & 1 & & & * \\
\hline
\end{tabular}

GE: Grupo ecológico - adaptado de CHAMI (2008) e ${ }^{\mathrm{I}} \mathrm{MOSCOVICH}$ (2006); P: pioneira; $\mathrm{S}_{\mathrm{i}}$ : secundária inicial; $\mathrm{S}_{\mathrm{t}}$ : secundária tardia; FV: formas de vida; $1=$ árvore; 2 =arbusto; CS: chuva de sementes; BS: banco de sementes do solo; BP: banco de plântulas. 


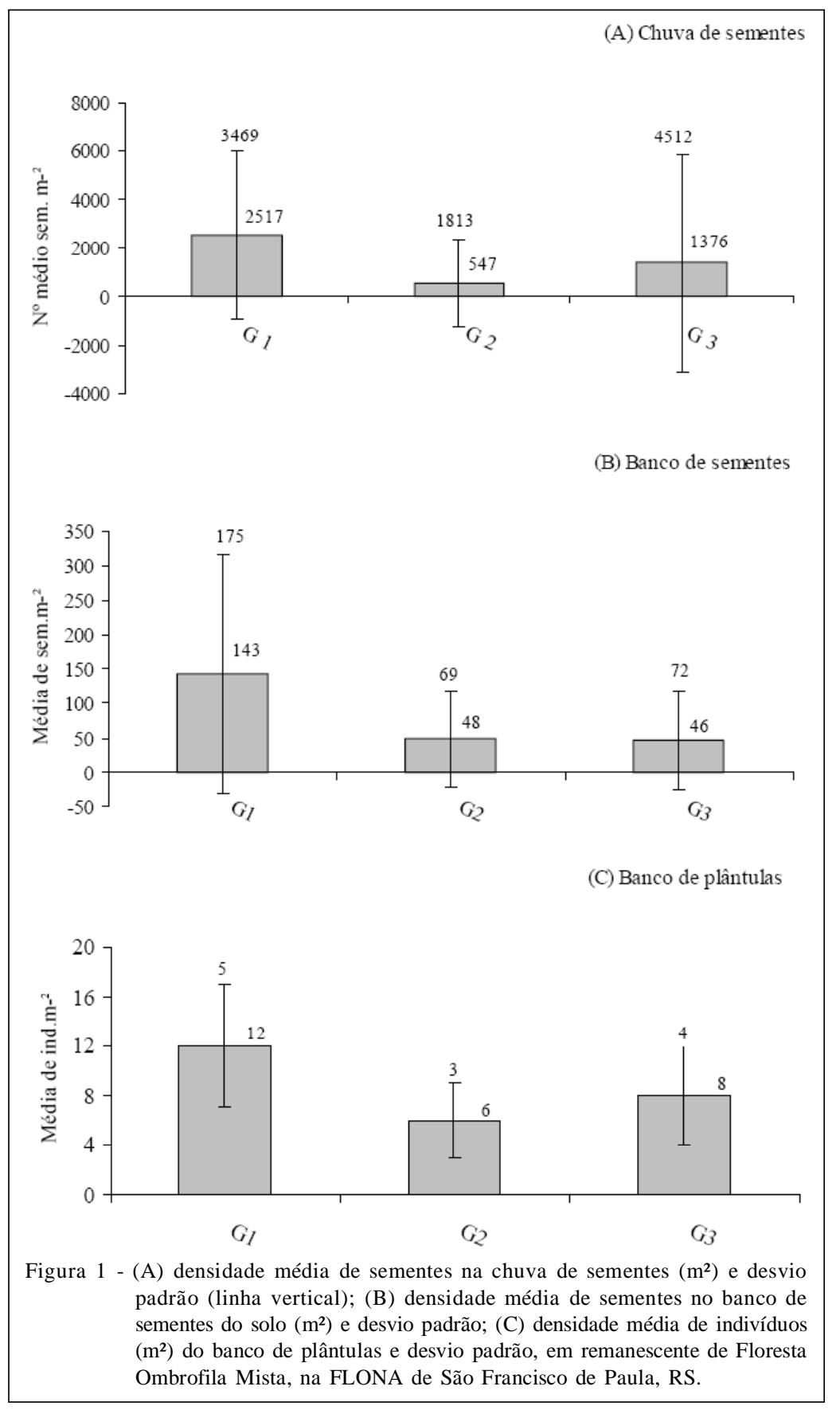

No banco de plântulas, as 72 unidades amostrais foram suficientes para representar as espécies presentes na regeneração natural na FLONA de São Francisco de Paula, uma vez que a curva espécieárea demonstrou tendência à estabilização com 63 subparcelas. No remanescente, foram encontradas 74 espécies de árvores e arbustos $(\mathrm{DAP}=1 \mathrm{~cm})$ de 28 famílias, entre as quais se destacaram Myrtaceae, Lauraceae, Fabaceae e Sapindaceae.
O número de indivíduos arbóreos e arbustivos no banco de plântulas apresentou diferença entre os três grupos. $\mathrm{O}_{1}$ possuiu a maior densidade de indivíduos, seguido do $\mathrm{G}_{3}$ e $\mathrm{G}_{2}$, respectivamente (Figura 1C). O maior potencial de regeneração observado no $G_{1}$ pode estar relacionado com o histórico de perturbação desse trecho, que, até aproximadamente 1980, teve intensa extração de madeira e, provavelmente, ainda esteja com elevada dinâmica de sucessão. 
Tabela 3 - Densidade das espécies arbóreas e arbustivas presentes com maior destaque nos mecanismos de regeneração dos distintos agrupamentos florísticos da Floresta Ombrofila Mista, na FLONA de São Francisco de Paula, RS.

\begin{tabular}{|c|c|c|c|c|c|c|c|c|c|}
\hline \multirow{2}{*}{ Nome científico } & \multicolumn{3}{|c|}{------------D $\mathrm{D}_{\mathrm{A}} \mathrm{BP}-\mathbf{- - - - - - - - - -}$} & \multicolumn{3}{|c|}{-----------D $\mathrm{D}_{\mathrm{A}} \mathrm{CS}$----------- } & \multicolumn{3}{|c|}{--- } \\
\hline & $\mathrm{G}_{1}$ & $\mathrm{G}_{2}$ & $\mathrm{G}_{3}$ & $\mathrm{G}_{1}$ & $\mathrm{G}_{2}$ & $\mathrm{G}_{3}$ & $\mathrm{G}_{1}$ & $\mathrm{G}_{2}$ & $\mathrm{G}_{3}$ \\
\hline Araucaria angustifolia & 417 & 69 & 208 & 2 & 3 & $*$ & & & \\
\hline Blepharocalyx salicifolius & 833 & 1250 & 2500 & $*$ & & & & & \\
\hline Cupania vernalis & 2708 & 694 & 2813 & 1,2 & $*$ & $*$ & & & \\
\hline Eugenia uruguayensis & 208 & 278 & 1458 & & & 2 & & & \\
\hline Ilex brevicuspis & & 764 & 1250 & 70 & 221 & 1200 & 34 & 11 & 25 \\
\hline Ilex microdonta & 208 & & & 597 & & & & & \\
\hline Ilex paraguariensis & 208 & 417 & 208 & 108 & 258 & 36 & 42 & 9 & 1 \\
\hline Lamanonia ternata & 1042 & 69 & & 200 & & * & 2 & & 1 \\
\hline Matayba elaeagnoides & 5625 & 2986 & 11458 & $*$ & 1 & $*$ & & & \\
\hline Mollinedia elegans & 52083 & 27847 & 6979 & & & & & & \\
\hline Myrceugenia cucullata & 625 & 972 & 3021 & & & & & & \\
\hline Myrceugenia oxysepala & 833 & 3472 & 5313 & & & & & & \\
\hline Myrsine coriacea & 417 & 486 & 208 & & & & 1 & 4 & 2 \\
\hline Myrsine laetevirens & & & & 70 & $*$ & & 6 & & \\
\hline Myrsine sp. & & & 313 & 3 & 3 & 4 & & & \\
\hline Myrsine umbellata & 5208 & 2431 & 2604 & & & & 1 & 3 & \\
\hline Podocarpus lambertii & 208 & & & 1 & & & & & \\
\hline Rudgea parquioides & 2917 & 833 & 1458 & 3 & & 1 & & & \\
\hline Sapium glandulosum & & & & $*$ & $*$ & 7 & 2 & $*$ & 4 \\
\hline Sebastiania brasiliensis & 417 & 833 & 1354 & & 36 & 25 & & & \\
\hline Sebastiania commersoniana & 1458 & 1806 & 2500 & $*$ & 4 & 29 & & & 2 \\
\hline Siphoneugena reitzii & 8958 & 625 & 4375 & & & & 7 & & \\
\hline Solanum mauritianum & & & & & & & 13 & 1 & \\
\hline Stillingia oppositifolia & 5000 & 2708 & 21458 & & & & & & \\
\hline Vernonia discolor & 417 & & & 861 & 9 & $*$ & & & \\
\hline Zanthoxylum rhoifolium & 1042 & & 208 & & & & 12 & 6 & 2 \\
\hline
\end{tabular}

$D_{A} B P$ : densidade de ind. banco de plântulas (n. de ind ha $\left.{ }^{-1}\right) ; D_{A} C S$ : densidade de sementes na chuva de sementes $\left(n\right.$. de sem $\left.m^{-2}\right) ; D_{A} B S$ : densidade de sementes no banco de sementes $\left(\mathrm{n}\right.$. de sem $\mathrm{m}^{-2}$ ); * valor $<1$.

No banco de plântulas, foram destaque Mollinedia elegans, Stillingia oppositifolia, Matayba elaeagnoides, Myrceugenia oxysepala, Siphoneugena reitzii, Myrsine umbellata, Sebastiania brasiliensis, Cupania vernalis, Blepharocalyx salicifolius, Myrceugenia cucullata, Rudgea parquioides e Casearia decandra. A maioria das espécies observadas no banco de plântulas foi classificada como secundárias tardias e clímax (Tabela 1 e 2) e, segundo KAGEYAMA e VIANA (1991), apresentam sementes que não necessitam de luz para a germinação e nem para o crescimento inicial da plântula. Os mesmos autores também descrevem o banco de plântulas como estratégia principal de regeneração das espécies desses grupos ecológicos. A predominância de espécies clímax e secundárias tardias no banco de plântulas da FLONA, portanto, sugere que esse remanescente encontra-se em estágio sucessional avançado.
A espécie melhor representada no banco de plântulas, M. elegans, foi descrita por PEIXOTO et al. (2001) como um arbusto de autoecologia esciófita ou de luz difusa. Já $\boldsymbol{C}$. decandra, $\boldsymbol{S}$. oppositifolia, $\boldsymbol{S}$. brasiliensis, B. salicifolius, M. elaeagnoides, $M$. umbellata, $R$. parquioides $\boldsymbol{e}$ M. cucullata foram consideradas por NARVAES et al. (2005) como predominantes na regeneração natural estabelecida na FLONA. Isso indica que essas espécies estão crescendo sob as condições de baixa luminosidade no estrato inferior da floresta. $\mathrm{O}$ recrutamento para as classes de maior tamanho, porém, está associado ao comportamento de cada espécie em relação à exigência de luz (heliófilo) e tolerância à sombra (esciófilo).

Considerando que das 86 espécies observadas na vegetação adulta (CHAMI, 2008), 51 formaram um banco de plântulas, foi possível sugerir que esse mecanismo seja a estratégia principal de manutenção dessas espécies na floresta. $\mathrm{No}_{1}$, foram 
destaques M. elegans, S. reitzii, M. elaeagnoides, $M$. umbellata e R. parquioides; no $\mathrm{G}_{2}, M$. elegans, $M$. oxysepala, M. elaeagnoides e S. oppositifolia; e, no $\mathrm{G}_{3}$, S. oppositifolia, M. elaeagnoides, M. elegans, $M$. oxysepala, S. reitzii, M. cucullata e C. vernalis (Tabela 3).

Com base nas espécies indicadoras observadas na vegetação adulta da FLONA (CHAMI, 2008), nas espécies abundantes na chuva de sementes, no banco de sementes e no banco de plântulas verificadas no presente trabalho, foram descritas como as espécies prováveis de se perpetuarem na FLONA, ao longo dos processos de sucessão: Araucaria angustifolia, Siphoneugena reitzii Ilex brevicuspis, Mollinedia elegans, Podocarpus lambertii e Vernonia discolor, em ambientes de encosta; Araucaria angustifolia, Casearia decandra, Blepharocalyx salicifolius, Mollinedia elegans, Ilex paraguariensis e Sebastiania brasiliensis, em ambiente de árvores emergentes; Araucaria angustifolia, Blepharocalyx salicifolius, Cryptocarya aschersoniana, Stillingia oppositifolia, Ilex brevicuspis, Sebastiania commersoniana e Siphoneugena reitzii, em terrenos de maior umidade.

\section{CONCLUSÃO}

A chuva de sementes foi abundante e representou uma fonte expressiva de propágulos para abastecer o banco de sementes e banco de plântulas local e das áreas circunvizinhas; o banco de sementes do solo não possui potencial florístico para substituir as espécies arbóreas e arbustivas presentes na vegetação, mas as espécies herbáceas predominantes e as poucas arbustivo-arbóreas pioneiras irão contribuir para restauração ecológica da área, caso ocorra perturbação intensa na floresta; e o banco de plântulas, em razão da riqueza de espécies, pode ser o mecanismo principal de manutenção da vegetação arbórea na FLONA de São Francisco de Paula.

\section{REFERÊNCIAS}

ALMEIDA-CORTEZ, J.S. Dispersão e banco de sementes. In: FERREIRA, A.G.; BORGHETTI, F. (Org.). Germinação: do básico ao aplicado. Porto Alegre: Artmed, 2004. 323p.

ARAUJO, M.M. et al. Caracterização da chuva de sementes, banco de sementes do solo e banco de plântulas em Floresta Estacional Decidual Ripária, Cachoeira do Sul, RS, Brasil. Scientia Forestalis, n.66, p.128-141,dez. 2004. Disponível em: <http://www.ipef.br/PUBLICACOES/SCIENTIA/nr66/ cap13.pdf>. Acesso em: 10 set. 2008.

BRAUN-BLANQUET, J. Fitossociologia: bases para el estudio de las comunidades vegetales. 3.ed. Madrid: H. Blume, 1979. 820 p.
BUDOWSKI, G.N. Distribution of tropical American rain forest species in the light of sucesión processes. Turrialba, v.15, n.2, p.40-52, 1965.

CARNELUTTI FILHO, A.C. et al. Testes não paramétricos para pesquisas agrícolas. Santa Maria: UFSM/CCR/ Departamento de Fitotecnia, 2001. 87p.

CHAMI, L.B. Vegetação e mecanismos de regeneração natural em diferentes ambientes da Floresta Ombrófila Mista na FLONA de São Francisco de Paula, RS. 2008. 126f. Dissertação (Mestrado em Ciências Florestais) Universidade Federal de Santa Maria, RS.

DUARTE, L.S. et al. Assessing the role of light availability in the regeneration of Araucaria angustifolia (Araucariaceae). Australian Journal of Botany, v.50, n.6, p.741-751, 2002.

EMBRAPA. Centro Nacional de Pesquisa de Solo. Sistema brasileiro de classificação de solos. Brasília: EMBRAPA SPI, 1999. 412p.

FERRETTI, A.R. Fundamentos ecológicos para o planejamento da restauração florestal. In: GALVÃO, A.P.M.; MEDEIROS, A.C. de S. (Ed.). A restauração da Mata Atlântica em áreas de sua primitiva ocorrência natural. Colombo: Embrapa Florestas. 2002. p.35-43.

FINEGAN, B.; SABOGAL, C. El desarrolo de sistemas de produccion sostenible en bosque tropical humedos da Bajura: un studio de caso en Costa Rica (1 $1^{\mathrm{a}}$ parte). El Chasqui, v.17, p.3$21,1988$.

HYATT, L. Differences between seed bank composition and fiel recroltment in a temperate zone deciduos forest. American Midland Naturalist, v.142, p.31-38, 1999.

KAGEYAMA, P.Y.; VIANA, V.M. Tecnologia de sementes e grupos ecológicos de espécies arbóreas tropicais. In: SIMPÓSIO BRASILEIRO SOBRE TECNOLOGIA DE SEMENTES FLORESTAIS, 2., 1989, Atibaia, SP. Anais... Atibaia: Instituto Florestal, 1991. p.197-215.

LEITE, P.F.; KLEIN, R.M. Vegetação. In: IBGE. Geografia do Brasil-Região Sul. Rio de Janeiro: IBGE, Fundação Instituto Brasileiro de Geografia e Estatística, 1990. p.113150.

LORENZI, H. Árvores brasileiras: manual de identificação e cultivo de plantas arbóreas nativas do Brasil. Nova Odessa: Plantarum, 2002. V.1-2, 384p.

MARTINS, S.V. et al. Banco de sementes como indicador de restauração de uma área degradada por mineração de caulim em Brás Pires, MG. Revista Árvore, v.32, n.6, p.1081-1088, 2008. Disponível em: <http://www.scielo.br/pdf/rarv/v32n6/ a13v32n6.pdf>. Acesso em 10 jun. 2010. doi: 10.1590/S010067622008000600013 .

MORENO, J.A. Clima do Rio Grande do Sul. Porto Alegre: Secretaria da Agricultura, 1961. 42p.

MOSCOVICH, F.A. Dinâmica de crescimento de uma Floresta Ombrófila Mista em Nova Prata, RS. 2006. 130f. Tese (Doutorado em Engenharia Florestal) - Universidade Federal de Santa Maria, RS. 
NARVAES, I.S. et al. Estrutura da regeneração natural em Floresta Ombrófila Mista na Floresta Nacional de São Francisco de Paula, RS. Ciência Florestal, v. 15, n.4, p.331-342, 2005. Disponível em: <http://www.ufsm.br/cienciaflorestal/artigos/ v15n4/A1V15N4.pdf>. Acesso em: 28 maio, 2009.

NIMER, E. Clima. In: IBGE. Geografia do Brasil- Região Sul. Rio de Janeiro: IBGE. Fundação Instituto Brasileiro de Geografia e Estatística, 1990. p.151-187.

PEIXOTO, A.L. et al. Monimiáceas. In: REITZ, R.; REIS, A. (Eds.). Flora ilustrada catarinense. Itajaí: Herbário Barbosa Rodrigues, 2001. 64p.
RIO GRANDE DO SUL. Secretaria Estadual do Meio Ambiente. Relatório Final do Inventário Florestal Contínuo do Rio Grande do Sul. Porto Alegre: SEMA/UFSM, 2001. 706p.

SCOTTI, M.S.V. Mecanismos de regeneração natural em remanescente de Floresta Estacional Decidual. 2009. 106f. Dissertação (Mestrado em Ciência Florestal) Universidade Federal de Santa Maria, RS.

VIEIRA, I.C.G. Forest succession after shifting cultivation in eastern Amazonia. 1996. 205f. Thesis (Doctor of Philosophy) - University of Stirling, Scotland. 